\section{La Declaración de Naciones Unidas sobre Derechos de los Pueblos Indígenas}

José Zalaquett Daher*
Este comentario, junto a los

documentos que analiza,

está disponible en

www.anuariocdh.uchile.cl

\begin{abstract}
Aparentemente, cuando la Asamblea General de las Naciones Unidas aprobó la Declaración sobre los Derechos de los Pueblos Indígenas ${ }^{1}$, el 13 de septiembre de 2007, se dio un paso cardinal hacia el fortalecimiento de la normativa internacional sobre esta materia. En este comentario se sostiene que efectivamente se trata de un avance significativo, pero con algunas importantes salvedades.
\end{abstract}

Al proclamar dicha declaración, la Asamblea General recogió la recomendación formulada por el Consejo de Derechos Humanos de las Naciones Unidas el año anterior ${ }^{2}$. Tal como se señala en un artículo publicado en la edición del año 2007 de este Anuario 2007³, la creación, en 2006, de dicho Consejo, que vino a reemplazar a la Comisión de Derechos Humanos de Naciones Unidas ${ }^{4}$, suscitó en su momento reacciones de esperanza y de escepticismo. Las últimas se basaban en la comprobación de que la composición de este nuevo órgano continuaba incluyendo a varios Estados con un historial de serias violaciones de los derechos humanos, así como la circunstancia de que durante los primeros tiempos de su funcionamiento, el Consejo parecía continuar aplicando, en varios casos, criterios políticos que con frecuencia rigieron las decisiones de la comisión que lo precedió. Por su parte, entre las razones para abrigar esperanzas se contaba el hecho de que se trataba de un nuevo cuerpo, dotado del estatus más elevado de órgano subsidiario de la Asamblea General, así como la circunstancia de que, tan pronto comenzó a trabajar, aprobó los textos de sendas declaraciones sobre, respectivamente, la desaparición forzada y los derechos de los pueblos indígenas.

En muchos sentidos, la Declaración de las Naciones Unidas sobre los Derechos de los Pueblos Indígenas constituye un progreso normativo. Para algunos, es un avance de mucha importancia. José Aylwin declara, en un artículo reciente, que se trata de "un hito de gran significación en la construcción de un consenso internacional sobre derechos humanos, en este caso, sobre los derechos de pueblos históricamente negados y discriminados, los que están integrados por más de 300 millones de personas en todo el planeta" ${ }^{5}$. En el mismo artículo, el autor reproduce la declaración

\footnotetext{
* Abogado, Licenciado en Ciencias Jurídicas y Sociales por la Universidad de Chile. Doctor en Derecho, Honoris Causa, por las universidades de Notre Dame y City University of New York. Miembro de la Comisión Interamericana de Derechos Humanos (2002 y 2005) y Presidente (2003 y 2004). Es profesor de Derechos Humanos de la Facultad de Derecho de la Universidad de Chile y Co-Director del Centro de Derechos Humanos de la misma casa de estudios.

1 Resolución A/61/ 295, adoptada en la 107ª sesión plenaria y distribuida con fecha 2 de octubre de 2007.

2 Resolución 1/2 del Consejo de Derechos Humanos de 29 de junio de 2006.

3 El Nuevo Consejo de Derechos Humanos de Naciones Unidas, Morton H. Halperin y Diane F. Orentlicher, Anuario de Derechos Humanos 2007, http://www.anuariocdh.uchile.cl/anuario03/6-seccionInternacional/anuario03_sec_ internacionalIV_HalperinyOrentlicher.pdf

4 Luego de 60 años de funcionamiento, la Comisión de Derechos Humanos de Naciones Unidas celebró su $62^{\text {a }}$ y última sesión el 24 de marzo de 2006, procediendo a traspasar sus asuntos y archivos al Consejo de Derechos Humanos.

5 "La Declaración de Naciones Unidas sobre Derechos de los Pueblos Indígenas y sus implicancias para América Latina", José Aylwin, artículo publicado en el sitio Web del Observatorio de Derechos de los Pueblos Indígenas [ www.observatorio. cl Consultado en enero de 2008].
} 
del Relator Especial de las Naciones Unidas para los Derechos Humanos y Libertades Fundamentales de los Indígenas, Rodolfo Stavenhagen, en el sentido de que "la Declaración refleja el consenso internacional cada vez más extendido en torno al contenido de los derechos indígenas...".

Con todo, como se fundamentará en el curso de este comentario, hasta donde es posible vislumbrarlo, es dudoso que esta declaración Ilegue a tener el efecto seminal de otras declaraciones de Naciones Unidas sobre derechos humanos, en el sentido de dar inicio a un proceso de generación normativa que culmine, años más tarde, en un instrumento internacional de carácter vinculante.

Por otra parte, mientras en el ámbito de las Naciones Unidas se llevaban a cabo, a lo largo de dos décadas, esfuerzos por producir la declaración que aquí se comenta, paralelamente se desarrollaban en América, a nivel local y regional, postulados académicos y, en especial, campañas, acciones y litigios en pro del reconocimiento de los derechos de los pueblos indígenas. Este proceso condujo, en varios países de la región, así como en el sistema interamericano de protección de los derechos humanos, a distintas reformas constitucionales, estándares jurisprudenciales y políticas públicas. Con todas sus insuficiencias, los mencionados desarrollos han contribuido y podrán seguir contribuyendo al progreso sostenido, aunque penosamente lento, del reconocimiento y respeto de los derechos de los pueblos indígenas en esta región. Quizás uno de los principales méritos de la declaración de Naciones Unidas objeto de este análisis, es que recoge y sistematiza estos avances. Por ello, puede constituirse en una referencia normativa que permita que en el futuro se alcancen mayores logros por las mismas vías de las campañas, acciones y litigios, las proposiciones doctrinarias, los estándares de la jurisprudencia interamericana, las reformas legales a nivel nacional y las políticas públicas.

En lo que sigue, se analizan las siguientes materias: (i) El contenido y la fuerza normativa de la Declaración de Naciones Unidas sobre derechos de los Pueblos Indígenas; (ii) las principales consideraciones políticas que explican algunos aspectos del texto final y los votos en contra que se dieron en el seno de la Asamblea General, al decidirse el proyecto de resolución; y, (iii) los avances en materia de estándares normativos que se han dado en el último tiempo, en el ámbito del sistema interamericano de protección de los derechos humanos.

\section{El Contenido y Fuerza Normativa de la Declaración de Naciones Unidas sobre Derechos de los Pueblos Indígenas}

El conjunto de derechos de los pueblos indígenas, según se ha ido conformando en las últimas décadas al calor de los desarrollos doctrinarios, jurisprudenciales y prácticos señalados en la sección anterior, suele agruparse en tres grandes categorías:

(i) Derechos colectivos a la libre determinación o a un estatus político de una autonomía tal que permita a estos pueblos afirmar y preservar su identidad, autogobernarse en importante medida, incluyendo la administración de justicia en ciertas materias, de acuerdo a sus propias costumbres ancestrales, y decidir o participar en la elaboración y ejecución de estrategias y planes de desarrollo para su comunidad.

(ii) Derechos colectivos a la tierra y recursos naturales, comprendiéndose entre estos últimos (al tenor de las proposiciones más recientes) los recursos pesqueros y del subsuelo, así como la protección del medio ambiente y de la biodiversidad de sus territorios.

(iii) Derechos relativos a la conservación y desarrollo de su cultura, lo que incluye, entre otros aspectos, el uso, preservación y/o difusión de su historia, idiomas, tradiciones, filosofías y religiones, medicina tradicional, artes y deportes. 
Como corolario de los derechos colectivos mencionados se agrega que los pueblos indígenas tienen derecho a contar con los medios para financiar sus funciones autónomas y que deben ser consultados en materias de leyes, políticas públicas y medidas gubernamentales que puedan afectar tales derechos.

A más de los derechos anteriores, de naturaleza colectiva, se añade que los indígenas, en cuanto individuos, son titulares de todos los derechos humanos, incluyendo los derechos políticos y el derecho a la nacionalidad, en el Estado dentro del cual habitan.

Todos estos derechos colectivos e individuales están reconocidos en detalle en la declaración de Naciones Unidas que aquí se comenta, con algunas omisiones y salvedades cuya razón se explica más adelante. Más aún, este nuevo instrumento internacional abunda en especificaciones que pudieran parecer evidentes o redundantes. Esta circunstancia requiere de una digresión, a fin de explicar la tradición de Naciones Unidas en lo que se refiere a declaraciones sobre los derechos humanos de determinados colectivos, conjuntos o categorías de personas, también llamados (controvertidamente) derechos de especificación.

Para comenzar, cabe recordar que para las Naciones Unidas una declaración de la Asamblea General es un tipo de resolución y, por tanto, estrictamente hablando, tiene el valor de una recomendación para los Estados, no una fuerza vinculante. Sin embargo, la declaración es un ejemplo muy especial de resolución. En efecto, se la considera "un instrumento solemne, que se utiliza sólo en casos muy especiales, de grande y verdadera importancia, y cuando se espera obtener el máximo de observancia por parte del mayor número de Estados posible" ${ }^{\prime 6}$.

El primer caso -y el más señero- de una resolución de Naciones Unidas que se ajusta a esta definición es la que la Asamblea General aprobó el día 10 de diciembre de 1948, conteniendo la Declaración Universal de Derechos Humanos. Existe amplio consenso internacional en el sentido de que esta declaración ha adquirido, por vía consuetudinaria, fuerza vinculante, aunque para los Estados que son parte del Pacto Internacional de Derechos Internacionales y Políticos y del Pacto Internacional de Derechos Económicos, Sociales y Culturales, ambos de 1966 (y que son la mayoría de los Estados) se hace innecesario invocar el carácter vinculante de la Declaración Universal, en la medida en que los mencionados Pactos desarrollan, por lo general en mayor detalle, los derechos que aquélla consagra.

Más adelante, partiendo con la Declaración de los Derechos del Niño, de 1959, se comenzó a suceder, a lo largo de los años, una serie de proclamaciones de Naciones Unidas sobre los derechos llamados de especificación. En la mayoría de los casos tales declaraciones se producían luego de proposiciones y demandas de determinados grupos y organizaciones las cuales terminaban por encontrar eco en ciertos Estados y en órganos de Naciones Unidas. A su vez, alcanzado el hito de una declaración, luego se abría paso, por lo general, a un proceso que conducía, años más tarde, a la aprobación de una convención internacional sobre la misma materia ${ }^{7}$.

6 www.un.org/spanish/

7 Por ejemplo, la Declaración de los Derechos del Niño data de 1959 y la Convención sobre los Derechos del Niño, de 1989. La Declaración sobre la eliminación de la discriminación contra la mujer es de 1967 y la Convención sobre la eliminación de todas las formas de discriminación contra la mujer, de 1979. Ocasionalmente, el tiempo que media entre una declaración y la convención respectiva es muy breve, si las circunstancias políticas y la voluntad de los Estados así lo determinan; así sucedió con la Declaración de las Naciones Unidas sobre la eliminación de todas las formas de discriminación racial, de 1963, que fue seguida dos años más tarde por la Convención Internacional sobre la Eliminación de todas las Formas de Discriminación Racial. El caso de la Convención internacional sobre la protección de los derechos de todos los trabajadores migratorios y sus familiares, de 1990, es excepcional entre los instrumentos de derechos humanos sobre los derechos de determinados colectivos o categorías de personas, en el sentido de que no fue precedida de una declaración. 
Por tanto, si bien una declaración de Naciones Unidas puede calificarse, dado que estrictamente carece de fuerza vinculante, de soft law o "derecho blando", habitualmente da impulso a un proceso que culmina en una convención internacional vinculante. Más aún, en la práctica, los derechos y estándares proclamados en una declaración determinada comienzan a invocarse desde ya por organizaciones no gubernamentales y movimientos de derechos humanos en sus campañas en pro del respeto de determinados derechos, independientemente de su carácter jurídico de soft law; del mismo modo, las declaraciones contribuyen al desarrollo de opiniones doctrinarias y, por esa y otras vías, influyen en los razonamientos y decisiones de tribunales nacionales y de las cortes u otros órganos de protección de los derechos humanos, sean internacionales o regionales. Como se verá más adelante, tal ha sido el caso en relación con los informes y sentencias generadas dentro del sistema interamericano de protección de los derechos humanos en lo que toca a peticiones concernientes a derechos de los pueblos indígenas.

Retornando al punto en que se comenzó esta digresión, cabe recalcar que las declaraciones de las Naciones Unidas sobre los Ilamados derechos de especificación suelen incluir afirmaciones que parecen innecesarias porque constituyen un corolario evidente de otras normas generales sobre derechos humanos. De hecho, es una opinión relativamente difundida en la literatura sobre los derechos humanos que, estrictamente hablando, los derechos de las mujeres, por ejemplo, no serían sino la aplicación a las mujeres de los derechos humanos generales; incluso normas tan específicas como la que señala que no son contrarias al principio de no discriminación las medidas afirmativas de carácter temporal que busquen superar una situación social, institucional, cultural y/o histórica de menoscabo de la mujer, podrían derivarse del mismo principio de no discriminación y de la obligación positiva de los Estados de garantizar y promover los derechos humanos. Tales opiniones se han vertido también a propósito de los derechos de los pueblos indígenas ${ }^{8}$.

Esas posiciones pueden tener fundamento desde un punto de vista académico. Sin embargo, el propósito de mucho del contenido de las declaraciones o convenciones sobre estas materias es no sólo jurídico, sino también político y educativo. En efecto, en la práctica no cabe esperar que los Estados o incluso los mismos órganos internacionales de protección de los derechos humanos, extraigan de principios y normas ya establecidos todo lo que lógicamente debe desprenderse de ellos en lo que dice relación con los derechos de colectivos o categorías de personas tradicionalmente discriminadas, marginadas o reducidas a la condición de relativa invisibilidad social.

No obstante lo anterior, es cierto también que las declaraciones de Naciones Unidas sobre derechos humanos con frecuencia incluyen juicios que parecen ir demasiado lejos en tratar de hacer explícito lo que es obvio. Por ejemplo, el artículo 7.2. de la declaración que aquí se comenta establece que los pueblos indígenas "...no serán sometidos a ningún acto de genocidio..." 9 .

La Declaración de Naciones Unidas sobre Derechos de los Pueblos Indígenas establece para los Estados obligaciones negativas, consistentes en no discriminar o en respetar (no transgredir) otros

8 En su artículo citado anteriormente (ver nota al pie 5) José Aylwin consigna la opinión del jurista indígena estadounidense James Anaya de que la Declaración de Naciones Unidas sobre Derechos de los Pueblos Indígenas "no crea derechos nuevos sino que reconoce a los indígenas derechos relativos a los pueblos y a las personas actualmente vigentes en el derecho internacional de los derechos humanos pero que hasta ahora no les han sido respetados en muchos de los Estados en que habitan".

9 Se puede argumentar que es necesario recordar que los pueblos indígenas, en muchas latitudes, han sido víctimas de genocidio a lo largo de la historia, pero ello bien podría haberse cumplido con una referencia en el preámbulo de la Declaración. La razón de la fórmula adoptada por la Declaración debe buscarse en la preferencia de los Estados por referirse al genocidio de los indígenas dentro de una norma prescriptiva y no mediante la afirmación de un hecho histórico (en el preámbulo), lo cual podría dar pie a demandas de reconocimientos o reparaciones a nivel nacional. 
derechos fundamentales de los pueblos indígenas, así como obligaciones positivas de garantizar (prevenir que los derechos respectivos se vean afectados, reparar y resarcir la violación de los mismos, incluyendo la restitución, cuando ello sea posible, y promover tales derechos, comprendido en ello la facilitación de recursos para su efectiva realización) y de consultar a los pueblos indígenas en materias que les conciernen.

Dada la naturaleza jurídica de las declaraciones, la que comentamos no contempla propiamente un órgano o mecanismo de protección. No obstante, en su artículo 42 señala que "Las Naciones Unidas, sus órganos, incluido el Foro Permanente para las Cuestiones Indígenas y los organismos especializados, en particular a nivel local, así como los Estados, promoverán el respeto y la plena aplicación de las disposiciones de la presente Declaración y velarán por la eficacia de la presente Declaración".

\section{Las Consideraciones Políticas}

Por lo general, de las tres categorías principales de derechos de los pueblos indígenas señaladas en párrafos anteriores, los Estados en cuyos territorios habitan estos pueblos encuentran menos dificultades en aceptar los de carácter cultural, mayores problemas en reconocer efectivamente los derechos relativos a la tierra y recursos naturales, y abrigan muy serias renuencias en lo relativo a los derechos colectivos de libre determinación o estatus político de autonomía.

La razón de esta diferencia es clara. El primer grupo de derechos puede requerir de los Estados y de la respectiva sociedad cambios culturales, reformas y recursos, pero, en términos generales, no plantean conflictos de intereses insuperables, sean económicos o políticos. El derecho a la tierra genera, en cambio, en muchos casos, la necesidad de enfrentar la recuperación o compensación territorial de dominios largamente arrebatados a los pueblos indígenas, los que se encuentran actualmente en poder de otros propietarios o en los cuales se han dado desarrollos urbanos; $y$ en lo que se refiere a los recursos naturales, los derechos de los pueblos indígenas suelen chocar con los del Estado respectivo o los de inversionistas o concesionarios interesados en explotarlos. Finalmente, el derecho de libre determinación o el derecho a un estatus de autonomía política levantan el espectro del debilitamiento de la soberanía, la fragmentación del Estado o de "una nación dentro de otra nación".

Las resistencias recién mencionadas son, por lo general, infundadas. Por una parte, hay ejemplos de países que han enfrentado (aunque imperfectamente) los derechos de libre determinación sin que se produjeran los efectos temidos. Por otra, si bien una solución que realice efectivamente los derechos indígenas requiere de cuantiosos recursos (por ejemplo, para la recuperación de tierras o el otorgamiento de tierras alternativas) ello constituye un imperativo de justicia. Con todo, no puede ignorarse que tales resistencias existen, aunque por lo común no son admitidas abiertamente, e influyen en la posición de los Estados respecto del reconocimiento internacional de los derechos de los pueblos indígenas ${ }^{10}$.

Por cierto que hay numerosos Estados para los cuales el voto a favor de declaraciones de las Naciones Unidas o la ratificación de convenciones internacionales no significa gran cosa, en la medida en que sus respectivos sistemas políticos y jurídicos no tienen el grado de desarrollo necesario

10 Un ejemplo de ello es la Declaración que sobre esta materia se ha venido negociando por largos años en el seno de la OEA. Este comentarista, a quien correspondió desempeñarse como Relator para los Derechos de los Pueblos Indígenas en el seno de la Comisión Interamericana de Derechos Humanos, llegó al convencimiento de que de parte de la mayoría de los Estados del sistema no existía real voluntad de avanzar en los trabajos preparatorios, pero tampoco estaban dispuestos a asumir el costo político de reconocerlo abiertamente. 
para que tales compromisos se adopten con suficiente seriedad y las normas respectivas tengan efectiva aplicabilidad en el derecho interno. Sin embargo, cada vez les resulta más arduo a los Estados sostener dicha actitud, debido a la presión creciente de organismos no gubernamentales especializados y de sectores de opinión (incluso dentro de los mismos países de que se trata), de órganos intergubernamentales de protección de los derechos o de otros Estados.

Por estas y otras razones semejantes, los Estados que han decidido adoptar planes y medidas más o menos sistemáticas para dar satisfacción a ciertas de las demandas de los pueblos indígenas de sus territorios, por lo general optan por tratar estos temas como un asunto interno.

El ejemplo del Convenio 169 de la Organización Internacional del Trabajo, de 1989 sobre Pueblos Indígenas y Tribales en Países Independientes (que reemplazó al convenio 107, de la misma organización de 1957), único instrumento internacional vinculante sobre la materia, pareciera desmentir la afirmación anterior. Si bien a la fecha sólo lo han ratificado 19 Estados, entre ellos se cuentan los siguientes países latinoamericanos a los cuales ciertamente les atañen sus disposiciones: Argentina, Bolivia, Brasil, Colombia, Costa Rica, Ecuador, Guatemala, Honduras, México, Paraguay, Perú y Venezuela ${ }^{11}$. No obstante que la ratificación del Convenio 169 por estos países ha tenido algún efecto interno, incluso en citas ocasionales por parte de tribunales nacionales, subsiste, en los hechos, la tendencia a manejar el "problema indígena" principalmente a partir de negociaciones, planes, leyes y medidas de carácter interno, independientemente del grado en que éstas se ajusten a las obligaciones que estipula el Convenio 169.

Las mismas motivaciones de orden político y el mismo deseo de manejar el tema de los derechos indígenas como asunto interno explican que los cuatro votos en contra de la Declaración provinieran de Australia, Canadá, Estados Unidos y Nueva Zelanda. Todos estos países cuentan con numerosas poblaciones indígenas y han adoptado leyes, acuerdos, políticas y planes internos en relación con las demandas y derechos de estos pueblos (cualquiera sea el juicio que merezca el mérito o justicia de tales iniciativas). Al mismo tiempo, todos ellos son países con instituciones jurídicas y políticas fuertes dentro de las cuales podría tener resonancia especial el hecho de que el respectivo Estado adhiera a determinados instrumentos internacionales.

Las motivaciones reseñadas en esta sección explican también que Declaración de Naciones Unidas sobre Derechos de los Pueblos Indígenas no se haga cargo de los conflictos que pueden surgir entre los derechos colectivos de los pueblos indígenas y los derechos humanos de individuos indígenas, o bien entre los derechos colectivos de los pueblos indígenas y los intereses y prerrogativas del Estado. Esta característica es común a las declaraciones sobre derechos humanos, así como a muchas convenciones que establecen obligaciones positivas de parte de los Estados. El texto de estos instrumentos tiende a acomodar los intereses y posiciones de los Estados, aunque la redacción resultante sea vaga, contradictoria o suponga la existencia de un plano ideal en el que las contradicciones se concilian. Sin embargo, en materia de derechos de los pueblos indígenas los conflictos entre distintos bienes jurídicos tienden a ser más marcados, en razón de la naturaleza colectiva de estos derechos y su contraposición potencial con intereses particulares o estatales.

Los siguientes son algunos de los conflictos potenciales que pueden presentarse, sin considerar el más problemático de todos, el relativo a la soberanía y jurisdicción: (i) individuos indígenas pueden ver afectados los derechos humanos que poseen de acuerdo a la Constitución del país respectivo o al derecho internacional, debido a la aplicación del derecho tradicional indígena o debido a una decisión de una autoridad comunitaria respecto de una situación que los afecta;

11 Al tiempo de terminar de escribir este comentario, con fecha 5 de marzo de 2008, el Senado de Chile aprobó la ratificación del Convenio 169. (La Cámara de Diputados la había aprobado en abril de 2000). 
ello puede ocurrir en materias como los derechos políticos, el derecho a no ser discriminado, el derecho de propiedad, el derecho al debido proceso o el derecho a no sufrir tratos o castigos crueles, inhumanos o degradantes $^{12}$; (ii) la propiedad colectiva de la tierra plantea problemas en relación con los sistemas legales existentes en el país en cuestión, en cuanto al registro, delimitación, uso, goce y disposición de la propiedad, además de cuestiones relativas a servidumbres legales que afecten a los inmuebles, propiedad minera y concesiones de explotación, entre otros; (iii) las leyes de un determinado país así como los tratados internacionales sobre preservación del medio ambiente pueden entrar en conflicto con las tradiciones indígenas, por ejemplo, respecto de la caza tradicional de especies protegidas.

Las situaciones específicas de conflicto que podrían presentarse son tan numerosas y complejas que probablemente no resultaría factible abordarlas en forma pormenorizada en una declaración o convención. Sin embargo, por razones fundamentalmente políticas, entre ellas el deseo de evitar los problemas inherentes al hecho de plantear estos problemas, los Estados han preferido no formular siquiera un reconocimiento general del tipo de conflictos que podrían presentarse junto con entregar alguna guía general sobre cómo enfrentarlos.

Hace excepción a lo dicho lo relativo al conflicto potencial entre libre determinación de los pueblos indígenas y soberanía nacional que, como queda señalado, es el tema de mayor preocupación para muchos Estados. Así, el artículo 46, número 1 de la Declaración establece: “Nada de lo señalado en la presente declaración se interpretará en el sentido de que confiere a un Estado, pueblo, grupo o persona derecho alguno a participar en una actividad o realizar un acto contrario a la Carta de las Naciones Unidas, ni se entenderá en el sentido de que autoriza o fomenta acción alguna encaminada a quebrantar o menoscabar, total o parcialmente, la integridad territorial o la unidad política de Estados soberanos e independientes".

\section{Avances normativos sobre derechos de los pueblos indígenas, dentro del sistema interamericano de protección de los derechos humanos}

Los informes de la Comisión Interamericana de Derechos Humanos y las sentencias de la Corte Interamericana de Derechos Humanos sobre casos concernientes a derechos de los pueblos y personas indígenas han aumentado significativamente en años recientes.

Algunos de estos casos tienen que ver con masacres en contra de personas o poblados indígenas ${ }^{13}$. En tales litigios la Corte Interamericana ha hallado que se ha violado el derecho a la vida y derechos conexos. No se ha tratado, por tanto, de derechos de los pueblos indígenas, sino de derechos humanos individuales transgredidos en gran escala mediante asesinatos masivos, aun cuando en su perpetración haya influido la condición de indígenas de las víctimas. Otro caso concerniente a la transgresión de derechos humanos generales pero que afecta a una población indígena es Yatama Vs. Nicaragua ${ }^{14}$. En este caso se trata específicamente de la violación de derechos políticos,

\footnotetext{
12 La Declaración, con todo, indica una regla muy general en su artículo 46 número 2: "En el ejercicio de los derechos enunciados en la presente Declaración se respetarán los derechos humanos y libertades fundamentales de todos. El ejercicio de los derechos establecidos en la presente Declaración estará sujeto exclusivamente a las limitaciones determinadas por la ley y con arreglo a las obligaciones internacionales en materia de derechos humanos. Esas limitaciones no serán discriminatorias y serán sólo las estrictamente necesarias para garantizar el reconocimiento y respeto debidos a los derechos y libertades de los demás y para satisfacer las justas y más apremiantes necesidades de una sociedad democrática" .

13 Entre los ejemplos más señeros se cuentan el Caso Masacre Plan de Sánchez Vs. Guatemala, Sentencia de 29 de abril de 2004. Serie C No 105 y el Caso de la comunidad Moiwana Vs. Suriname, Sentencia del 15 de junio de 2005. Serie $\mathrm{CN}^{\circ} 124$.

14 Sentencia de 23 de Junio de 2005. Serie C No 127.
} 
no en el sentido de la libre autonomía de los pueblos indígenas, sino en el significado de que los partidos políticos que representan a estas comunidades puedan postular candidatos a elecciones realizadas dentro del Estado que habitan, sin restricciones ni discriminaciones indebidas.

Los informes y sentencias del sistema interamericano que abordan propiamente derechos de los pueblos indígenas se han concentrado mayoritariamente en el derecho colectivo a la tierra, con algunas referencias al derecho a recursos naturales y a la protección del medio ambiente.

La Comisión Interamericana de Derechos Humanos emitió dos informes en esta materia sobre casos presentados en contra de Estados Unidos y de Belice, respectivamente. Dado que estos países no son parte de la Convención Americana sobre Derechos Humanos, el marco normativo en que se basó la Comisión fue el de la Declaración Americana de los Derechos y Deberes del Hombre, de 1948, además de las normas convencionales y estatutarias que otorgan a la Comisión la facultad y el deber de velar por la protección y promoción de los derechos humanos respecto de los países miembros de la OEA.

En el primero de dichos casos, el de Mary y Carrie Dann, presentado contra Estados Unidos ${ }^{15}$, la Comisión concluyó que si bien la cuestión que conoció "involucra aspectos complejos de derecho y de hecho que competen más a la determinación del Estado... estos procesos deben conformarse con las normas y principios de la Declaración Americana aplicables a la determinación de los derechos de propiedad de los indígenas conforme se describen en este informe".

En el segundo de dichos casos, el de la Comunidad Indígenas Mayas del Distrito de Toledo, presentado contra Belice ${ }^{16}$, la Comisión concluyó que "el Estado violó el derecho a la propiedad consagrado en el artículo XXIII de la Declaración Americana y el derecho a la igualdad consagrado en el artículo II de la Declaración Americana, en perjuicio del pueblo maya, al no adoptar medidas efectivas para delimitar, demarcar y reconocer oficialmente el derecho de propiedad comunal a las tierras que han ocupado y usado tradicionalmente, y por otorgar concesiones madereras y petroleras a terceros, para utilizar los bienes y recursos que podrían estar comprendidos dentro de las tierras que deben ser delimitadas, demarcadas y tituladas, sin consultar al pueblo maya ni obtener su consentimiento informado". También concluyó que el Estado violó el derecho a la protección judicial consagrado en el artículo XVIII de la Declaración Americana en perjuicio del pueblo Maya.

Los informes precedentemente citados se emitieron con posterioridad al caso Awas Tingni ${ }^{17}$, la principal sentencia de la Corte Interamericana de Derechos Humanos sobre derechos colectivos a la propiedad de la tierra de los pueblos indígenas, la cual es citada en el informe sobre la petición de las Comunidades Indígenas Mayas del Distrito de Toledo.

En Awas Tingni, la Corte Interamericana determinó que Nicaragua violó el artículo 21 de la Convención Americana sobre Derechos Humanos que establece el derecho de toda persona al uso y goce de sus bienes. A este respecto declaró lo siguiente: "Mediante una interpretación evolutiva de los instrumentos internacionales de protección de derechos humanos, tomando en cuenta las normas de interpretación aplicables y, de conformidad con el artículo 29.b de la Convención -que prohíbe una interpretación restrictiva de los derechos-, esta Corte considera que el artículo 21 de la Convención protege el derecho a la propiedad en un sentido que comprende, entre otros, los

\footnotetext{
15 Caso 11.140, Informe de Fondo $N^{\circ}$ 75/02, de 27 de diciembre de 2002.

16 Caso 12.053, Informe de Fondo № 40/04 de 12 de octubre 2004.

17 Caso de la Comunidad Mayagna (Sumo) Awas Tingni Vs. Nicaragua. Sentencia de 31 de agosto de 2001. Serie C $N^{\circ} 79$.
} 
derechos de los miembros de las comunidades indígenas en el marco de la propiedad comunal, la cual también está reconocida en la Constitución Política de Nicaragua"18.

La Corte resolvió que el Estado debe delimitar, demarcar y titular la propiedad colectiva del pueblo Mayagna.

Más tarde, en dos casos contra Paraguay, Yakye Axa ${ }^{19}$ y Sawhoyamaxa ${ }^{20}$, la Corte abundó en similares consideraciones sobre el derecho de las comunidades reclamantes a la propiedad colectiva de la tierra pero agregó muy interesantes fundamentos sobre cómo la circunstancia de hallarse ambas comunidades en situación paupérrima y de abandono, debido a la falta de reconocimiento de sus derechos a la tierra (con todo lo que ello implicaba en cuanto a su dignidad, sustento y cultura), representaba una violación de su derecho a la vida.

Así, la Corte determinó que el caso Yakye Aksa debía establecer si "el Estado generó condiciones que agudizaron las dificultades de acceso a una vida digna de los miembros de la Comunidad Yakye Axa y si, en ese contexto, adoptó las medidas positivas apropiadas para satisfacer esa obligación, que tomen en cuenta la situación de especial vulnerabilidad a la que fueron llevados, afectando su forma de vida diferente (sistemas de comprensión del mundo diferentes de los de la cultura occidental, que comprende la estrecha relación que mantienen con la tierra) y su proyecto de vida, en su dimensión individual y colectiva, a la luz del corpus juris internacional existente sobre la protección especial que requieren los miembros de las comunidades indígenas, a la luz de lo expuesto en el artículo 4 de la Convención, en relación con el deber general de garantía contenido en el artículo 1.1 y con el deber de desarrollo progresivo contenido en el artículo 26 de la misma, y de los artículos 10 (Derecho a la Salud); 11 (Derecho a un Medio Ambiente Sano); 12 (Derecho a la Alimentación); 13 (Derecho a la Educación) y 14 (Derecho a los Beneficios de la Cultura) del Protocolo Adicional a la Convención Americana en materia de Derechos Económicos, Sociales y Culturales, y las disposiciones pertinentes del Convenio No 169 de la OIT"21.

Como se advierte de los fallos citados en esta sección, la Corte Interamericana de Derechos Humanos ha ido aplicando a los casos de demandas de derechos de los pueblos indígenas que ha conocido, los conceptos, estándares y normas desarrollados en la evolución de la doctrina sobre esta materia, así como los que brotan, lo que llama el corpus juris internacional sobre la protección especial que requieren los miembros de las comunidades indígenas.

\section{Conclusión}

La Declaración de Naciones Unidas sobre Derechos de los Pueblos Indígenas, en la medida en que recoge y sistematiza (con algunos bemoles) el estado actual de las proposiciones sobre reconocimiento de tales derechos, contribuirá a aclarar e incluso a reforzar el corpus juris internacional sobre la materia. Sin embargo, como las propias sentencias de la Corte Interamericana de Derechos Humanos citadas en la sección anterior confirman, en definitiva la resolución de las cuestiones de asignación de recursos, de precisiones jurídicas y de conciliación de distintos intereses y derechos que inevitablemente trae aparejadas el reconocimiento efectivo de los derechos de los pueblos indígenas, se librará en el ámbito interno de los Estados.

\footnotetext{
18 Sentencia referida, párrafo 148.

19 Caso Comunidad indígena Yakye Axa Vs. Paraguay. Sentencia de 17 de junio de 2005. Serie C No 125.

20 Caso Comunidad Indígena Sawhoyamaxa Vs. Paraguay. Sentencia de 29 de marzo de 2006. Serie No C 146.

21 Sentencia Caso Yakye Aksa, párrafo 163.
} 
Por las razones antes indicadas en este comentario, no parece probable que haya nuevos desarrollos normativos internacionales, en el futuro previsible, que añadan precisión a estos derechos colectivos, entreguen directrices generales de cómo resolver los conflictos de derechos o intereses o establezcan mecanismos vinculantes de protección de estos derechos y de adjudicación de controversias. Con todo, los progresos normativos y jurisprudenciales en el orden internacional, aunque generales en su contenido, contribuyen a añadir peso a las demandas y procesos de negociación por el reconocimiento de estos derechos por parte de los pueblos indígenas y de las organizaciones y sectores que las apoyan. 